\title{
EL SOLDADO FANFARRÓN GASCÓN EN EL TEATRO FRANCÉS DEL XVI Y XVII
}

\author{
Josef PROKOP \\ Universidad de Bohemia del Sur, České Budějovice
}

\begin{abstract}
En): In the $16^{\text {th }}$ and $17^{\text {th }}$ century the braggart soldier receives a Spaniard identity in the Spanish and Italian theatre. Surprisingly French theatre assumes this tendency only partially. Generally its braggart soldiers appear without any geographical origin. It is only in the early $17^{\text {th }}$ century when the braggart begins to be depicted there as Gascon. This article aims to reflect on this phenomenon in comparison with the mentioned appearance of the Spanish braggart in Italy. On one hand, the literary life of the Spanish soldier is associated with the Spanish military and courteous prestige in $16^{\text {th }}$ century Europe and the character dies off gradually with the vanishing of the latter. The Gascon braggart on the other hand, experienced the biggest upswing after the peak period of Gascon military and social prestige in France. It is apparent that Spanish and Gascon braggarts on the stage are affected by uneven literary dynamics.
\end{abstract}

Key words (En): Braggart soldier; Alazón; Gascon identity; French theatre of the $16^{\text {th }}$ and $17^{\text {th }}$ century

Palabras claves (Es): soldado fanfarrón; alazón; identidad gascona; teatro francés del siglo XVI y XVII

En el teatro europeo de los siglos XVI y XVII habita el extraño personaje del soldado vanaglorioso y fanfarrón, cuyas reencarnaciones en este período son, por ejemplo, el capitán francés Matamore, el italiano Spaventa o simplemente il Capitano de la commedia dell'arte. No obstante, su genealogía es mucho más antigua de lo que parece a primera vista. Los gérmenes del personaje ya se encuentran en el más antiguo teatro griego y su más conocido precursor de la antiguiedad es Pirgopolinices de Miles gloriosus de Plauto. El protagonista renace para una nueva vida a principios del siglo XVI en el teatro español e italiano y en este « siglo español » se le imprime una característica muy específica - muchos de estos fanfarrones de la comedia italiana se convierten en mercenarios españoles (reales o imaginarios), nacionalizándose así como españoles.

Sin embargo, si esperáramos que este personaje se expandiera también por el dominio del reino francés, nos equivocaríamos. Y esto a pesar de que los cortesanos, los amantes y los soldados españoles sean en este momento muy admirados en Francia. Incluso se convierten en tema literario cuando algunos autores franceses recogen anécdotas sobre sus hechos y dichos como lo hace sieur de Brantôme en sus Rodomontades espaignolles con su primera redacción a finales del siglo XVI. No obstante, en el teatro francés, entre los personajes de fanfarrones que aparecen por primera vez en La reconnue de Rémy Bellau de 1564 y en Le Brave de 1567 de Jean-Antoine de Baïf no encontramos ningún fanfarrón español. Tanto más sorprendente es el hecho de que sea un fanfarrón gascón el que se una en cierto momento a los vanagloriosos soldados franceses.

En otro lugar hemos tratado de resumir el desarrollo del personaje teatral del fanfarrón y el debate sobre su nueva vida en España e Italia en el siglo XVI (PROKOP, en prensa). En las siguientes líneas trataremos de ofrecer algunas ideas, todavía tentativas y premonitorias en su gran mayoría, acerca de las circunstancias y motivos del nacimiento de un soldado fanfarrón gascón en el teatro y generalmente en la literatura francesa.

Echo des études romanes $I X / 2,2013 \quad$ - 59 -

ISSN 1804-8358 (Online) - www.eer.cz 
El origen y la génesis del personaje del fanfarrón en el antiguo teatro griego y latino han sido ya satisfactoriamente descritos. CRAWFORD (1911), BOUGHNER (1954), LidA DE MALKIEL (1957), Milani (1965) y DE Michele (1999) están esencialmente de acuerdo sobre el hecho de que el personaje deriva del arquetipo del alazón o jactancioso del antiguo teatro griego. Por primera vez aparece de forma cercana como el general Lámaco en Los Acarnienses de Aristófanes alrededor del año 425 antes de Cristo (ARISTÓFANES, 1991 : 32 y sqq) y luego reaparece en varias ocasiones en las piezas de Menandro como, por ejemplo, el soldado Bias de la comedia Kolax del autor mencionado (DE MiCHELE, 1999 : 910). Esta tradición griega posteriormente la recogen y desarrollan Plauto y Terencio y, en una de las comedias del primer mencionado, nace un fanfarrón ejemplar, Pirgopolinices de Miles gloriosus que se convertirá en referencia para todos los comediógrafos en Italia, Francia y España durante el Renacimiento tardío.

A continuación, el personaje no sale en tablas antes de principios del siglo XVI. La discusión de los expertos sobre la primacía de su nueva aparición sigue abierta. CRAWFORD (1911) y BOUGHNER (1940, 1943, 1954) consideran, como el punto de partida de un nuevo interés en este personaje, la comedia $I$ due felici rivali de Iacopo Nardi fechada aproximadamente en 1513, mientras que LIDA DE MALKIEL (1957) en una brillante recensión del libro de 1954 de Boughner y MiLANI (1965) declara como primer fanfarrón moderno al personaje llamado Centurio de las escenas añadidas a La Celestina. Esta ampliación suele designarse como la Tragicomedia de Calisto y Melibea y se fecha en el año 1502. De los defensores de la posición intermedia que consideran las dos vías de desarrollo del personaje como autóctonas e independientes, mencionaremos a Fausto DE MiCHELE (1999).

En cuanto al primer fanfarrón español en la comedia italiana, por mucho tiempo se ha considerado como tal el personaje de Giglio de la comedia Gli'Ingannati de 1531, firmada colectivamente por los Academici Intronati di Siena (BoughNER, 1943 : 51-52). Virgil I. MiLANI (1965 : 225-226), sin embargo, traslada la fecha aún más en la historia y señala como el primer fanfarrón español en el teatro italiano al personaje Castiglia (sic) de la pieza La Nutrice de Lorenzo di Filippo Strozzi, estrenada entre los años 1512 y 1519. De todos modos, parece evidente que la nacionalización española del carácter se produjo en Italia casi inmediatamente después de su nueva resurrección a principios del siglo XVI.

Las razones por las que es precisamente el soldado español el que se convierte en el fanfarrón típico y emblemático de la comedia italiana son bien conocidas. Por un lado, el lenguaje y las galanterías españolas se ofrecían directamente a ser ridiculizadas porque ya a principios del siglo XVI entraron en moda y fueron admiradas e imitadas por gran parte de la Europa refinada y aristocrática. Rafael Lapesa señala que la lengua española fue en ese momento, por lo menos en Italia, considerada como «latina sonante y elegantísima »(LAPESA, 1959: 191). Castiglione, de otro lado, considera a los españoles como maestros de costumbres galantes y Juan Valdés nos afirma que quien quiere ser valorado como delicado, aristocrático y cortés en Italia tiene que expresarse en castellano. Es el momento 
cuando Gil Vicente comienza a escribir en español y cuando toda Europa traduce o imita el Amadís, la Cárcel de amor, La Celestina o el Lazarillo (LAPESA, 1959 : 191-197). Los españoles son admirados por su elegancia que confina con el afeminamiento. Difunden en Europa el uso de perfumes, brazaletes, guantes y la constante caza a las mujeres (BOUGHNER, 1943 : 50). Una caricatura de este hombre de moda mediante el personaje del fanfarrón español es una reacción que parece absolutamente lógica y natural.

Por otro lado, es precisamente en este momento cuando se distinguen los mercenarios españoles con éxitos innegables en el oficio militar. No sólo dominaron una parte del continente americano, sino que también emprendieron campañas más o menos exitosas por toda Europa y gracias al poder de sus armas dominaron varias ciudades y regiones italianas. Por encima de todo, figuran como iniciadores y catalizadores de dos de los eventos más terribles del siglo en suelo italiano, nos referimos al sacco de Prato y al de Roma.

Todo esto es un contexto social e histórico muy propicio para sugerir la compensación de los propios fracasos militares de los italianos mediante la identificación del jactancioso pero cobarde soldado con el español (cfr. LIDA DE MALKIEL, 1957 : 281; BoughNER, 1943 : 50; De MiCHELE, 1999 : 7).

No es de sorprender entonces que los autores italianos, a menudo, definan como españoles incluso personajes teatrales cuyo contexto textual no lo admite en ningún sentido. Aludimos aquí, por ejemplo, a Lodovico Domenichi y su obra teatral Due corteggiane (1563) - una bastante servil imitación de Bacchides de Plauto - en la cual sustituye al personaje de Cleomaco por un capitán español llamado Martín Alonso (BOUGHNER, 1943 : 51).

No obstante, la fama teatral del fanfarrón español en Italia casi desaparece a principios del siglo siguiente. Mejor dicho, este personaje de apariencias muy dinámicas se transforma en uno rígido y estático, en una especie de máscara inmovible - típico de la commedia dell'arte italiana - que se limita a un repertorio repetitivo de chistes y gestos ya conocidos. De acuerdo con Fausto DE MiCHELE (1999 : 17), creemos que el motivo más importante para esta transformación del personaje en Italia fue el gradual desvanecimiento del prestigio de los españoles. Los soldados españoles desaparecieron de Europa expulsados por contraataques y sufrieron fundamentalmente también por el colapso de su economía. El lenguaje y la galantería españolas ya no suscitan tanto entusiasmo como al comienzo del siglo pasado. El español altivo y orgulloso, hasta hace pocos años omnipresente casi en toda Europa, desaparece y no puede servir de modelo e inspiración para otras potenciales variaciones de la figura del fanfarrón. Y por consiguiente, éste se convirtió en un mero recuerdo de su glorioso pasado, en una especie de esqueleto fosilizado después de que su cuerpo se pudriera.

La evolución del personaje del fanfarrón gascón en el teatro francés aparentemente podría seguir la misma lógica como la del fanfarrón español en Italia. Esto es, apoyarse en los efectos de la gloria militar y del relativo prestigio social y cortesano del que gozaron durante el reinado del rey navarrofrancés, Enrique de Navarra. Sin embargo, veremos que el fanfarrón gascón aparece en la comedia francesa muy tardíamente y además de forma encubierta. Es decir, que se le suele designar como gascón pero sus hechos y dichos, su lengua y 
su manera de hablar, no lo distinguen en la mayoría de los casos de un soldado de origen francés. Y esto, a pesar de que el personaje del soldado vanaglorioso gascón apareció en la literatura francesa a finales del siglo XVI y a principios del XVII en varias ocasiones.

Como ya se ha dicho, la figura del soldado fanfarrón surge en la comedia francesa por primera vez - casi medio siglo después de sus primeras apariciones italianas y españolas - en una paráfrasis de Miles gloriosus plautino denominado Le Brave, obra de Jean-Antoine de Baïf de 1567, cuya primacía podría cuestionar, según una parte de la crítica, solamente Rémy Belleau con La reconnue de 1564. De allí se propagan los soldados fanfarrones por la comedia francesa durante el resto del siglo XVI y al menos hasta el siglo siguiente. Dejemos de lado en este momento los conocidísimos avatares de este arquetipo que aparecen hasta en el siglo XIX - el capitán Fracasse de Théophile Gautier inspirado por el Roman comique de Paul Scarron o el famoso narizón Cyrano de Bergerac creado por Edmond Rostand, este último incluso con un énfasis significativo en la identidad gascona del protagonista.

El primer fanfarrón gascón en la literatura francesa, no obstante, aparece fuera del escenario y antes de las piezas de Baïf y Belleau. Se trata de Gratianauld de Saint Sever mencionado sólo episódicamente en el Tiers livre rablesiano de 1546 (RABELAIS, 1546 : 291-295). A pesar del reducido espacio de solo un capítulo, su figura aparece descrita de una manera muy animada y viva. De hecho, es un fanfarrón modelo, jugador de naipes y matón que no solo es designado en el texto como gascón sino que no usa otra lengua para comunicarse que la gascona. Robert LAFONT recuerda (1971b : 23-24) que en el uso de gascón - principalmente correcto - por Gratianauld no hay ninguna intención caricaturesca, puesto que éste se adapta de forma natural al concepto plurilingüístico que Rabelais mantiene en sus novelas gargantuescas. Gratianauld rablesiano nos queda por lo tanto como un modelo de fanfarrón gascón en estado puro.

Otro famoso fanfarrón gascón es el capitán Triboulet del extenso panfleto de Guillaume de Reboul llamado Actes du synode universel de la Saincte Réformation de 1599. Nuestro protagonista aparece solamente como uno de los oradores en el sínodo mencionado, no obstante, su comportamiento violento junto con su exuberancia verbal hacen de él otro fanfarrón modelo de las letras francesas. También en su caso la identidad gascona queda marcada no solo por una designación nominal sino también por su gascón algo macarrónico en el que formula su arenga.

La tercera aparición significativa y fundamental del fanfarrón gascón en la literatura francesa llega con el barón Faeneste de la novela dialogada Avantures du baron de Faeneste de Agrippa d'Aubigné, el primer y el segundo libro de la novela publicados en 1617 y el tercero en 1619 (D'AUBIGNÉ, 1969 : 1347). El interés general que levantó la novela (el último cuarto libro fue publicado en Ginebra en 1630) y la presencia de casi todas las características típicas del fanfarrón en un estado cristalino hacen de la historia un texto indispensable para nuestro análisis. D’Aubigné polemiza en su texto con el concepto de la vida cortesana y refinada como el arte de fingir propio significado y valor. Los dos principales protagonistas de la novela, el caballero Enay (« einai » 
en griego « ser ») y el barón Faeneste (« phainesthai » en griego « parecer ») personifican estas actitudes contradictorias. El barón Faeneste ya por su propio nombre está definido como un fanfarrón vanaglorioso, mientras que su interlocutor Enay encarna las cualidades consideradas por el autor como inherentes a la existencia ética y razonable. La identidad gascona queda patentada por varias referencias concretas y también a lo largo del libro por la obstinada manera de hablar de Faeneste en un francés cómico y mutilado por rasgos fonéticos del gascón. Un francés a la gascona que junto con otras características sirve para ridiculizar al personaje por sus posturas y origen. $\mathrm{Y}$ todo esto del puño de d'Aubigné, antiguo aliado y servidor del rey navarro y campeón de los gascones Enrique, llegado a ser el rey de Francia.

Faeneste, sin duda, representa una de las realizaciones más logradas del arquetipo de fanfarrón gascón en la literatura francesa. Como ya se ha dicho, la llegada del personaje al teatro se produjo un poco más tarde. Además, los protagonistas teatrales son fanfarrones completos con todas las características determinativas, no obstante su identidad gascona solo se manifiesta mediante la designación como tales. Es de notar que estos fanfarrones no hablan ni actúan de manera diversa a la de los fanfarrones franceses. No usan el gascón o, como Matamore de Corneille, se limitan a soltar un par de veces la conjura «Cadédiou! » sin nada más. En otras palabras, no se distinguen casi en ninguna manera del fanfarrón corriente del teatro francés.

Tal es el caso del ya mencionado capitán gascón Matamore de L'Illusion comique de Pierre Corneille de 1636, igualmente como el capitán epónimo de Le véritable capitaine Matamore ou Fanfarron de Antoine Mareschal fechado a 1640. La última pieza es curiosa también por otra coincidencia y esta es que el capitán Matamore aparece en una refundición declarada del Miles gloriosus platutiano y mientras que todos los otros personajes conservan sus nombres tradicionales griegos, el fanfarrón queda bautizado como Matamore - dicho de paso, un nombre cuya etimología apunta mucho más hacia el suelo ibérico con su reconquista que hacia Gascuña. No obstante, el nombre se petrifica en Francia como un asociativo indispensable del fanfarrón gascón y así se llama también el capitán Matamore en los diálogos teatrales de Paul Scarron Les boutades du Capitan Matamore del 1646.

Antes de que analicemos esta particular situación tenemos que plantearnos la cuestión de la existencia o no de una identidad gascona. ¿Existe algo así ya en el siglo XVI? Primero, habrá que resumir solo en grandes pinceladas el contexto histórico. La corona francesa trata ya desde la Guerra de los cien años de atar con lazos cada vez más estrechos las regiones vecinas que hasta entonces no fueron relacionadas sólo muy remotamente en la época carolingia. A principios del siglo XVI, este proceso llega a su fin y desde este momento el rey francés directamente gobierna casi todo el territorio que hoy conocemos como la República francesa. Y es precisamente la región del norte de los Pirineos la que supo mantener su independencia en esta época. A finales del siglo XV, gracias a la voluntad de los reyes de Francia, nace aquí una alianza política dispar creada por la unión del condado de Foix, de Bearn, de la parte septentrional del anterior reino de Navarra ibérico y de otras dependencias de la dinastía reinante de Albret (LAFONT, 1971a : 
51-54). Para nuestro tema no podemos olvidar que Bearn y Navarra seguían siendo, al menos de iure, estados soberanos y por ende independientes de la corona francesa. Los gobernantes de la mencionada dinastía Albret intentaron fabricar en sus dominios un estado centralizado moderno, cuyos atributos definitorios fueron el uso del local bearnés como la lengua oficial del Estado de un lado y la adopción del cristianismo reformado como la religión del estado. Este conglomerado de países no incluía a toda la Gascuña cuya gran parte pertenecía ya bastante firmemente a la corona francesa, pero a pesar de esto supo crear un sentimiento común bearneso-navarro que se convirtió en sentimiento de la identidad regional gascona.

La existencia de la conciencia de tal identidad puede documentarse no solo en los escritos de carácter oficial (LAFONT, 1971: 54), sino también en la literatura. Uno de los textos de esta índole, un poco tardío pero muy elocuente, es el poema ocasional Accueill de la Roine de Navarre compuesto por Salluste du Bartas para darle en 1578 la bienvenida a Margarita de Valois, hermana del rey francés Carlos IX a su entrada en la ciudad de Nerac, ciudad residencial de la corte del dominio navarro-bearnés. El contenido de este curioso poema trilingüe es la disputa de tres ninfas - una latina, otra francesa y otra gascona sobre el derecho de saludar a la nueva reina de Navarra. Las ninfas se expresan en sus propios idiomas, en el caso de la gascona la lengua no es el bearnés oficial sino el gascón de la llanura natal de Du Bartas. Después de una argumentación muy explicativa, las dos primeras ceden y por motivos de soberanía política y autonomía reconocen el derecho de la ninfa gascona. En este sentido, también metafóricamente, reconocen el concepto de una identidad « gascona » propia y diferente de la francesa o latina (PROKOP, 2003). Añadiremos que el autor del poema, Salluste du Bartas, en ese momento cortesano del rey navarro Enrique, es de Monfort (actual departamento de Gers) precisamente un gascón de una tierra perteneciente ya desde hace varios siglos a la corona francesa. Pasajes similares que se pronuncian explícitamente sobre la identidad gascona los encontramos también en otros textos de autores gascones de este tiempo. Otro ejemplo muy explicativo es la Epístola versificada de Peyre Garros (LAFONT, 1971a : 68-71).

Por lo tanto, a pesar de que aún no se había creado una entidad política independiente que abarcara todo el territorio y que una parte considerable de los gascones perteneciera por vía administrativa y jurídica a la jurisdicción de la corona francesa, la población entre los Pirineos y el valle del Garona era en su mayoría consciente de su identidad propia definida geográfica e históricamente. Si bien, podemos adelantar las deducciones finales del análisis, ya la aparición - sea sólo nominal - del fanfarrón gascón en las obras de autores franceses sugiere que esta identidad fue percibida y reconocida también por los franceses.

Tenemos que preguntarnos ahora, ¿funcionaron en el caso del fanfarrón gascón en Francia unos mecanismos de prestigio militar y social similares a los que ejercieron su influjo sobre los fanfarrones españoles en Italia? A primera vista parece que sí. Es interesante comprobar que la situación de los soldados gascones en Francia del siglo XVI compagina casi perfectamente con la situación de los españoles. 
La segunda mitad del siglo XVI es en Francia un período de agitación y de caos debido a las guerras de religión por un lado y a la crisis de sucesión al trono de los últimos Valois por otro. Los gascones tomaron parte muy activa en la solución de ambas. En el caso de las guerras de religión estuvieron presentes en los tres lados opuestos del conflicto - los hugonotes, la Liga Católica y el partido de los Valois. Es esencial destacar que en cualquiera de estos partidos mostraron el valor y la destreza militar que sobrepasaban en muchos casos las cualidades mostradas de otros y en el caso de algunos se convirtieron en soldados legendarios de su época. Típico campeón gascón entre los mercenarios que por su carrera militar, en parte pertenece incluso al período anterior a las guerras italianas, sería el famoso mariscal Blaise de Monluc que, gracias a sus memorias militares Commentaires (publicado póstumamente en 1592), entró hasta la literatura francesa. Asimismo, en este siglo tan varonil podemos encontrar más personajes similares. Citemos solo al duque d'Épernon o al personaje clave de la época, el rey Enrique de Navarra, futuro monarca francés, cuyos éxitos políticos y también militares le valieron el aura de héroe « nacional », en la extensión semántica de la palabra de aquel momento obviamente. Así lo afirman varias composiciones elogiosas en el momento de la muerte del rey «Enric » navarro-gascón. Entre ellos sobresale la monumental obra, el poema épico y caballeresco Lo Gentilome gascon compuesto por Guillaume Ader en 1610 que celebra las virtudes militares y cortesanas del difunto.

En la segunda mitad del siglo XVI, el origen gascón ya implicaba que el soldado fuera fuerte y valiente. Y al prestigio militar pronto se unió el prestigio social. Como resultado de las circunstancias conocidas se convirtió el mencionado Enrique de Navarra en 1589 en el rey de Francia, y cuando después de varios años finalmente consolida su poder real sobre el reino, a Louvre le acompaña una legión de seguidores y compañeros gascones. Gracias a los lazos personales y a la adhesividad connacional muchos consiguen un cargo o función rentables y con ellos un prestigio innegable. Sin embargo, al mismo tiempo, sus costumbres, su incomprensible gascón y su mutilación de la lengua francesa muy pronto se convertirán en el blanco de los chistes de los cortesanos hostiles.

Todo esto sucede después de 1594, el año de la conversión al catolicismo de Enrique. Sin embargo, el rey es asesinado en 1610 y se queda como regente su segunda esposa, María dei Medici, una italiana. Esto implica el final de los favores para los gascones y el comienzo de los tiempos de bonanza para los italianos.

Si comparamos esta cronología con la aparición del fanfarrón gascón en la literatura y los escenarios teatrales tal y como lo hicimos en el caso de los españoles en Italia, tenemos que concluir que los mecanismos utilizados anteriormente no se aplican en este caso.

El fanfarrón gascón aparece en las comedias francesas ya después del período de auge del prestigio de los soldados gascones en el centro del poder. Si el fanfarrón español desaparece de los escenarios teatrales después de que España y sus caballeros pasen de moda a finales del siglo XVI y principios del XVII y se mantiene solamente como una figura esquemática en la commedia dell'arte, el fanfarrón gascón de otro lado precisamente entra en la escena después de la muerte 
de su gran mecenas e ídolo, curiosamente cuando ya no le espera ningún prestigio social reconocido.

¿Cómo se podría explicar esta discrepancia? En primer lugar, por la imperfección de la analogía a la que nos referimos. La situación política y social de los soldados españoles en Italia no era idéntica en todos sus detalles a la del soldado gascón en Francia. Por otra parte, como ya se ha mencionado, el fanfarrón gascón entra en la escena de la comedia francesa bastante tardíamente, a pesar de nacer como una figura literaria casi cien años antes de su salida al escenario ideada por Pierre Corneille. Además, esta entrada en la escena es casi virtual puesto que el fanfarrón gascón de las comedias francesas no difiere mucho ni en cuanto a las características del personaje, ni en la conducta o estilo de expresión, por no hablar de la elección del lenguaje del fanfarrón francés o, mejor dicho, del fanfarrón no marcado en general. Ambos personajes profundizan los motivos jactanciosos y la vehemencia fingida de la espada que había conocido ya el alazón griego y que conocía también el antiguo soldado español.

Si dejamos de lado los pormenores de la difícil cuestión del mecanismo y de las condiciones de la entrada del fanfarrón gascón en el teatro francés, quedaremos ante un hecho indiscutible. Y esto es que los autores de las comedias francesas de la primera mitad del siglo XVII sintieron la necesidad - aunque sea sólo superficial y formal - de introducir en escena a un fanfarrón específicamente diseñado que no fuera francés sino gascón. Y si fue gascón, esto nos indica que tuvo que existir cierta reticencia hacia el prestigio y preeminencia de los gascones y que la ridiculización del gascón en la máscara de fanfarrón tuvo que ser, por lo menos en alguna parte, motivada por la necesidad de una compensación psicológica

\section{BIBLIOGRAFÍA}

\section{Estudios}

Boughner Daniel C. (1940), Don Armado and the Commedia dell'Arte, Studies in Philology 37, p. 201-224.

Boughner Daniel C. (1943), The Braggart in Italian Renaissance Comedy, PMLA 58, no. 1, p. 42-83.

BoughnER Daniel C. (1954), The Braggart in Renaissance Comedy, Minneapolis, The University of Minnesota Press.

CRAWFORD J. P. Wickersham (1911), The Braggart Soldier and the Rufián in the Spanish Drama of the Sixteenth Century, Romanic Review 2, p. 186-208.

DE MiCHELE Fausto (1999), Il guerriero ridicolo e la sua storia, ovvero dal comico sovversivo alla maschera vuota, Quaderni d'italianistica 20.1-2, p. 7-20.

LAFONT Robert (1971a), La Renaissance du Sud, Essai sur la littérature occitane au temps de Henri IV, Paris, Gallimard.

LAFONT Robert dir. (1971b), Le Sud et le Nord, Toulouse, Privat.

LAPESA Rafael (1959), Historia de la lengua española, 4a ed., Madrid, Escelicer.

LIDA DE MALKIEL María Rosa (1957), El fanfarrón en el teatro del Renacimiento, Romance philology 11, p. 268-291. 
MiLANI Virgil I. (1965), The Origins of the Spanish Braggart in Strozzi's Commedia Erudita, Italica 42, no. 3, p. 224-230.

PROKOP Josef (en prensa, previsto para 2014), El arquetipo de soldado fanfarrón aportación española a la literatura mundial, in: Valeš Miroslav y Demlová Jana, Héroe y antihéroe en las literaturas hispánicas, Liberec, Technická univerzita Liberec, p. 23-43.

ProKOP Josef (2003), Poema trilingüe Accueil de la Roine de Nauarre de Salluste Du Bartas como prueba de existencia de la identidad nacional gascona al final del siglo XVI, in: Codifications et symboles des cultures nationales, actes du colloque tenu du 13 au 15 juin 2002 à l'Université de Brno, Brno, Masarykova univerzita v Brně.

\section{Obras literarias}

ADER Guillaume (2010), Lo gentilòme gascon, Orthez, Per noste.

BAÏF Jean-Antoine de (1567), Le brave, Paris, Robert Estienne.

BELlEAu Rémi (1989), La reconnue, Genève, Droz.

CORNEILLE Pierre (1639), L'illusion comique, Paris, F. Targa.

D’AuBIGNÉ Agrippa (1969), Oeuvres, texte établi par H. Weber, Paris, Gallimard.

GAUTIER Théophile (1892), Le Capitaine Fracasse, Paris, Charpentier et Fasquelle.

MARESCHAL Antoine (1640), Le véritable capitan Matamore ou le Fanfaron, Paris, Toussainct Quinet.

MONTLUC Blaise de (1981), Commentaires, Paris, Gallimard.

RABELAIS François (1546), Tiers liure des faictz et dictz herö̈ques du noble Pantagruel, Paris, Chrestien Wechel.

Rostand Edmond (1991), Cyrano de Bergerac, Poitiers, Grimaud Lebeaux.

SCARRON Paul (1647), Les boutades du capitan Matamore et ses comédies, Paris, A. de Sommaville.

SCARRON Paul (1985), Le Roman comique, Paris, Gallimard. 